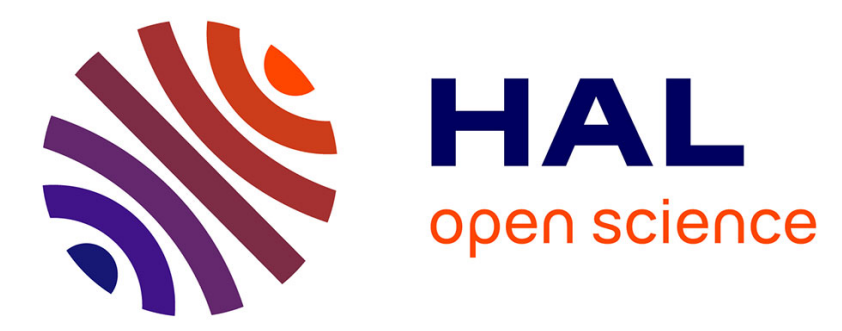

\title{
Numerical Simulation of Friction Stir Welding by Natural Element Methods
}

\author{
I. Alfaro, L. Fratini, Elías Cueto, Francisco Chinesta
}

\section{To cite this version:}

I. Alfaro, L. Fratini, Elías Cueto, Francisco Chinesta. Numerical Simulation of Friction Stir Welding by Natural Element Methods. International Journal of Material Forming, 2009, 2 (4), pp.225-234. 10.1007/s12289-009-0406-z . hal-01007130

\section{HAL Id: hal-01007130 \\ https://hal.science/hal-01007130}

Submitted on 19 Dec 2016

HAL is a multi-disciplinary open access archive for the deposit and dissemination of scientific research documents, whether they are published or not. The documents may come from teaching and research institutions in France or abroad, or from public or private research centers.
L'archive ouverte pluridisciplinaire HAL, est destinée au dépôt et à la diffusion de documents scientifiques de niveau recherche, publiés ou non, émanant des établissements d'enseignement et de recherche français ou étrangers, des laboratoires publics ou privés. 


\title{
Numerical Simulation of Friction Stir Welding by Natural Element Methods
}

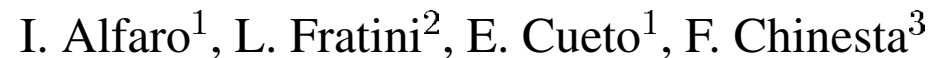

${ }^{1}$ I3A, University of Zaragoza, Zaragoza, Spain

URL: http://i3a.unizar.es_e-mail: iciar@unizar.es; ecueto@unizar.es

${ }^{2}$ Dept. of Mechanical Technology, Production and Management Engineering University of Palermo, Italy

URL: http://www.dtpm.unipa.it/mtg e-mail: fratini@dtpm.unipa.it

${ }^{3}$ Lab. de Mecanique des Systemes et des Procedes, LMSP UMR 8106 CNRS-ENSAM. Paris, France

URL: http://www.paris.ensam.fr/lmsp e-mail: francisco.chinesta@ paris.ensam.fr

\begin{abstract}
In this work we address the problem of numerically simulating the Friction Stir Welding process. Due to the special characteristics of this welding method (i.e., high speed of the rotating pin, very large deformations, etc.) finite element methods encounter several difficulties. Meshless methods somewhat alleviate this problems, allowing for an updated Lagrangian framework in the simulation. Accuracy is not affected by mesh distortion (and hence the name meshless), but the price to pay is the computational cost, higher than in the FEM. In this paper we will show some examples on the performance of the method.
\end{abstract}

KEYWORDS: Friction Stir welding, meshless methods, NEM

\section{INTRODUCTION}

Friction stir welding (FSW) is a solid state welding process used to join two pieces of sheet or plate material as aluminium, copper, lead and even some plastics. It achieves the welding of the pieces by employing a rotating pin and shoulder that provoke both extremely high plastic deformation and also a high heat generation. It is schematically represented in figure 1. At first, the sheets or plates are abutted along edge to be welded and the rotating pin is sunken into the sheets/plates until the tool shoulder is in full contact with the sheets or plates surface. Once the pin is completely inserted, it is moved with a small nutting angle in the welding direction. Due to the advancing and rotating effect of the pin and shoulder of the tool along the seam, an advancing side and a retreating side are formed and the softened and heated material flows around the pin to its backside where the material is consolidated to create a high-quality, solid-state weld.

In this paper we focus on the numerical simulation of the process, rather than the experimental characterization. From the numerical point of view, such a process presents several challenging difficulties. First of all, the extremely large deformations present dur- ing the process, although very localized in a region around the pin, always introduce numerical problems. Second, there is a strong coupling between these large deformations and heat generation, that in turn affects the behavior of the material. Last, it is important to notice the difficulty of simulating the FSW of two plates of different material.

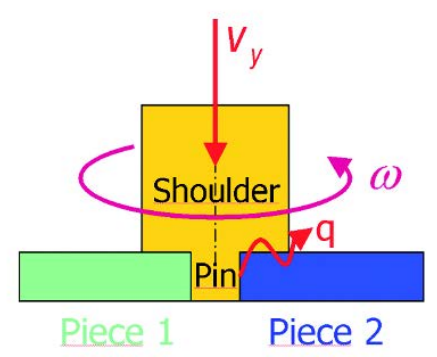

Figure 1: Schematic representation of the FSW process.

Up to our knowledge, few numerical attempts have been made in order to simulate this process. In [3] a FE based Lagrangian approach with intensive remeshing is employed. Similar approaches have been employed in [2]. Remeshing, however, is wellknown as a potential source of numerical diffusion in the results. In this paper we employ a somewhat different approach based on the use of meshless meth- 
ods. Meshless methods allow for a Lagrangian description of the motion, while avoiding the need for remeshing. Thus, the nodes, that in our implementation transport all the variables linked to material's history, remain the same throughout the simulation. Mappings between old and new meshes are not necessary and hence the avoidance of numerical diffusion.

Among the many meshless methods available nowadays, we have chosen the Natural Element Method [6] [4]. It possesses some noteworthy advantages over other meshless methods that will be described shortly.

\section{THE NATURAL ELEMENT METHOD}

Consider a model composed by a cloud of points $\boldsymbol{N}=\left\{n_{1}, n_{2}, \ldots, n_{m}\right\} \subset \mathbf{R}^{d}$, for which there is a unique decomposition of the space into regions such that each point within these regions is closer to the node to which the region is associated than to any other in the cloud. This kind of space decomposition is called a Voronoi diagram of the cloud of points and each Voronoi cell is formally defined as (see figure 2):

$$
T_{I}=\left\{\boldsymbol{x} \in \mathbf{R}^{d}: d\left(\boldsymbol{x}, \boldsymbol{x}_{I}\right)<d\left(\boldsymbol{x}, \boldsymbol{x}_{J}\right) \forall J \neq I\right\}
$$

where $d(\cdot, \cdot)$ is the Euclidean distance function.
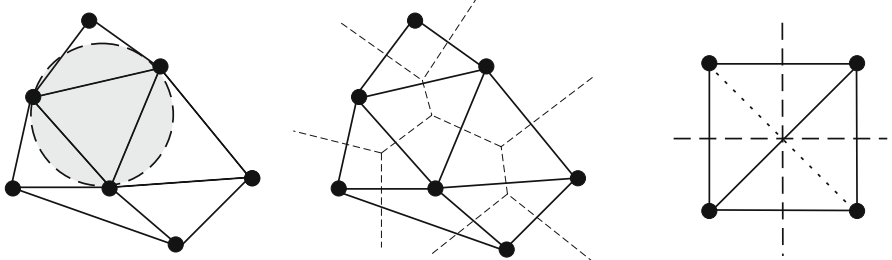

Figure 2: Delaunay triangulation and Voronoi diagram of a cloud of points.

The dual structure of the Voronoi diagram is the Delaunay triangulation, obtained by connecting nodes that share a common $(d-1)$-dimensional facet. While the Voronoi structure is unique, the Delaunay triangulation is not, there being some so-called degenerate cases in which there are two or more possible Delaunay triangulations (consider, for example, the case of triangulating a square in $2 \mathrm{D}$, as depicted in figure 2 (right)). Two nodes sharing a facet of their Voronoi cell are called natural neighbours and hence the name of the technique.
The most extended natural neighbour interpolation method is the Sibson interpolant [5]. Consider the introduction of the point $\mathrm{x}$ in the cloud of nodes. Due to this introduction, the Voronoi diagram will be altered, affecting the Voronoi cells of the natural neighbours of $\mathrm{x}$. Sibson [5] defined the natural neighbour coordinates of a point $\boldsymbol{x}$ with respect to one of its neighbours $I$ as the ratio of the cell $T_{I}$ that is transferred to $T_{x}$ when adding $\boldsymbol{x}$ to the initial cloud of points to the total volume of $T_{x}$.

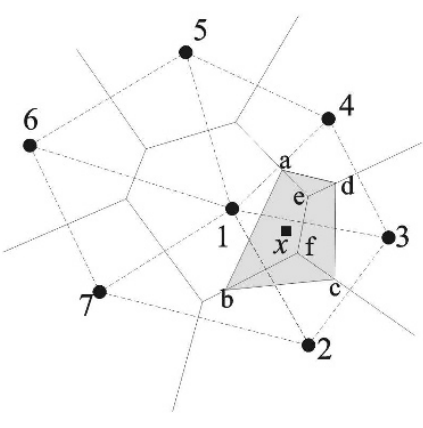

Figure 3: Definition of the Natural Neighbour coordinates of a point $\boldsymbol{x}$.

In figure 3 the shape function associated to node 1 at point $\mathrm{x}$ may be expressed as

$$
\phi_{1}(\boldsymbol{x})=\frac{A_{a b f e}}{A_{a b c d}}
$$

Sibson's interpolation scheme possesses the usual reproducing properties for this class of problems, i.e., verify the partition of unity property (constant consistency), linear consistency (and therefore are suitable for the solution of second-order PDE). This kind of interpolation scheme is then used in a Galerkin framework, exactly like in the finite element method.

\section{GOVERNING EQUATIONS}

FSW processes involve large deformation and high velocities of the rotating pin that make the elastic strains to be negligible. Although an elastic recovery exists, it is obvious negligible as a first approximation. The obvious advantage of this assumption is that the material can then be modeled as a non-newtonian (visco-plastic) fluid. This assumption is known as the flow formulation in the forming processes community [7]. Thus, the essential variables of the problem will be velocities and pressures, instead of displacements and pressures. 
The stresses will be, under this assumption,

$$
\boldsymbol{\sigma}=2 \mu \boldsymbol{d}-p \boldsymbol{I}
$$

where $p=-\operatorname{tr}(\boldsymbol{\sigma}) / 3$ and $\boldsymbol{I}$ stands for the secondorder identity tensor. The viscosity $\mu$ can depend on the strain, the strain rate, the temperature and other variables.

We consider, for the plates being welded, the balance of momentum equations without inertia and mass terms and the assumed incompressibility of a von Mises-like flow:

$$
\nabla \boldsymbol{\sigma}=0 \quad \nabla \boldsymbol{v}=0
$$

where $v$ represents the velocity field. Velocities are interpolated by means of the Sibson shape functions, while pressures are considered constant over the whole Voronoi cell associated to each node.

This rigid-plastic material is coupled with the following heat transfer equation:

$$
\rho c_{p} \frac{D T}{D t}=\nabla(k \nabla T)+\dot{r}
$$

where $k$ denotes the thermal conductivity, $\dot{r}$ the heat generation rate, $\rho$ the specific density and $c_{p}$ the specific heat of the metal. The rate of heat generation in the aluminium billet due to plastic deformation is given by $\dot{r}=\beta \boldsymbol{\sigma}: \boldsymbol{d}$ where $\beta$ represents the fraction of mechanical energy transformed to heat and is assumed to be 0.9 .

The coupling has been made through a blockiterative semi-implicit method, together with a fixedpoint algorithm to treat the non-linear coupling. This strategy has also been employed successfully in previous works of the authors [1].

\section{NUMERICAL RESULTS}

We consider here a very simplified two-dimensional plane stress model of the union of two plates with a rigid pin. Despite that the usual friction coefficient between the pin and the sheet has been established to be around 0.5 we have considered here perfect adhesion between them. This will overestimate the strain produced by the pin rotation. The material chosen for both plates of this first example corresponds to aluminium AA7075, with a viscosity, taken from [2]:

$$
\mu=\frac{K T^{A} d^{n-1}}{3}
$$

whith $K=2.69 \cdot 10^{10} \frac{\mathrm{N}}{\mathrm{mm}^{2}}, A=-3.3155, \mathrm{n}=$ 0.1324, and the following thermal properties: $k=$ $180 \frac{\mathrm{N}}{s K}$ and $\rho c_{p}=2.39 \frac{\mathrm{N}}{\mathrm{mm}^{2} \mathrm{~K}}$. The pin has a diameter of $3 \mathrm{~mm}$, rotates at $1000 \frac{\mathrm{rev}}{\mathrm{min}}$ and advances at $1 \frac{\mathrm{mm}}{\mathrm{s}}$.

In figure 4 the evolution of the temperature is shown, checking the good agrement with the FEM results shown in [2]. It is also checked that the properties of the material, such as the viscosity, change due to the temperature evolution (see figure 5).
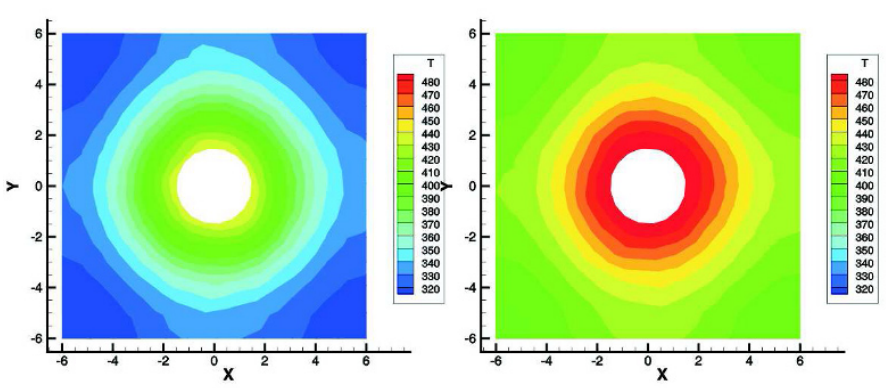

Figure 4: Temperature. Left: 100th time step.Right: 400th time step
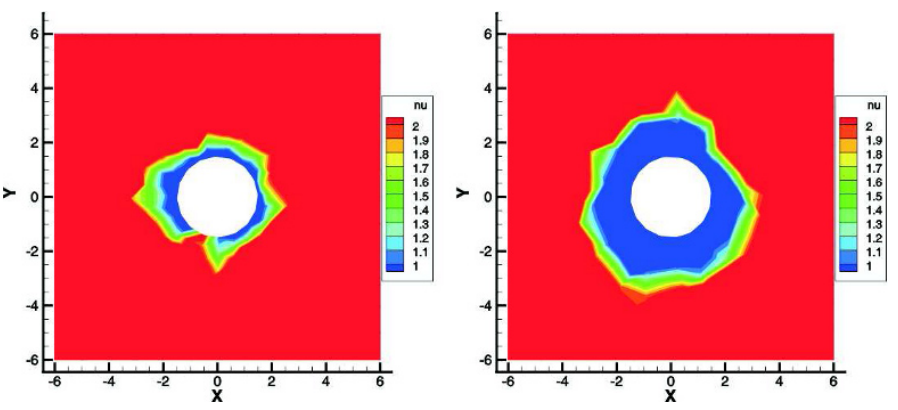

Figure 5: Viscosity. Left: initial time step. Right: 400th time step.

The most important advantage of the presented technique is the possibility of tracking the materials particles throughout the process. Now we have considered two plates of different materials, with viscosity defined by $\mu=K * d^{n-1}$. They have equal consistency parameter $K=1$ and different sensitivity index: $n=1$ and $n=0.6$. Now only rotating movement is studied, thus the advancing speed is zero.

In figures 6 to 8, several steps of the simulation are shown. Nodes of each plate have been labeled, by painting them in blue and red. This tracking can be performed without remeshing despite the high level of distortion of the resulting triangulation because triangles do not constitute the support of NEM shape 
functions. In this way, the numerical diffusion provoked by continuous remeshing is avoided. In addition, if some kind of nodal integration is performed, all history variables can be linked to the nodes, thus avoiding any projection procedures.

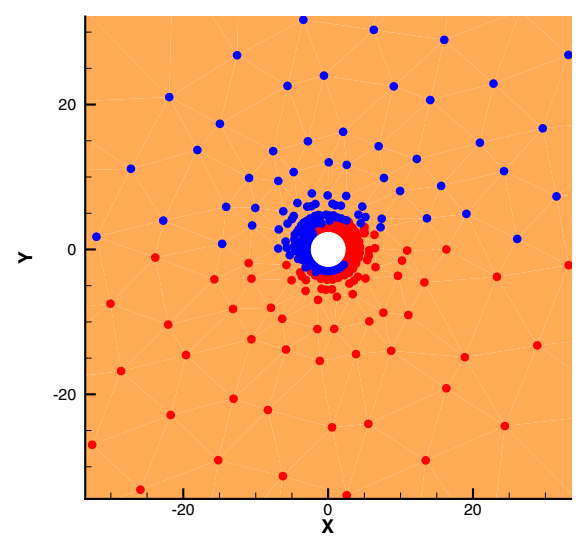

Figure 6: Material distribution at 50th time step.

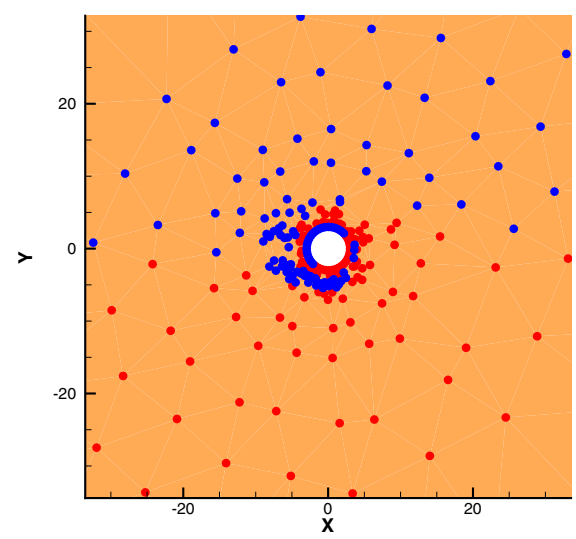

Figure 7: Material distribution at 150th time step.

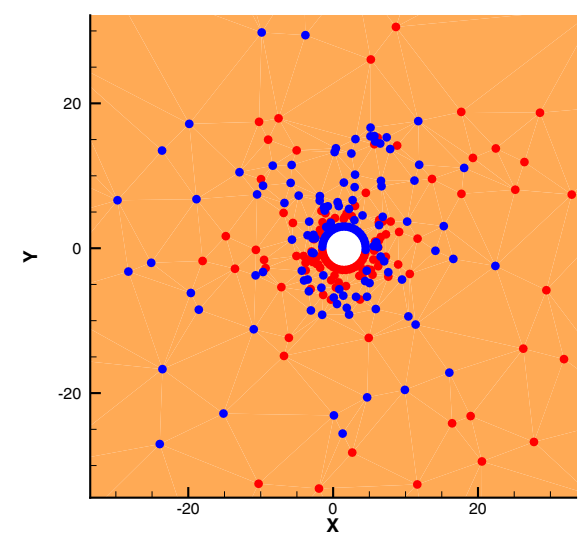

Figure 8: Material distribution at 6000th time step.

\section{CONCLUSIONS}

Although the presented results can be considered as very preliminary, and further refinement of the models (especially relative to contact and friction) is needed, we can conclude that the NEM constitutes a valuable tool for the simulation of such a complex forming process. In particular, it has been shown how a large number of time steps have been accomplished maintaining the initial set of nodes. Despite the high distortion of the triangulation, good qualitative agreement with previous results has been found. Note, however, that the true interest of the proposed technique relies in its extension to three-dimensional settings, where the true complexity of the process should be analyzed. This constitutes the ongoing work of the authors.

\section{REFERENCES}

[1] I. Alfaro, D. Bel, E. Cueto, M. Doblare, and F. Chinesta. Three-dimensional simulation of aluminium extrusion by the $\alpha$-shape based natural element method. Computer Methods in Applied Mechanics and Engineering, 195(3336):4269-4286, 2006.

[2] G. Buffa, J. Hu, R. Shivpuri, and L. Fratini. A continuum based fem model for friction stir welding-model development. Materials Science and Engineering A, 419:389-396, 2006.

[3] J.-L. Chenot and E. Massoni. Finite element modelling and control of new metal forming processes. Journal of Machine Tools and Manufacture, 46:1194-1200, 2006.

[4] E. Cueto, N. Sukumar, B. Calvo, M. A. Martnez, J. Cegoino, and M. Doblare. Overview and recent developments in Natural Neighbour Galerkin methods. Archives of Computational Methods in Engineering, 10 (4):307-384, 2003.

[5] R. Sibson. A Vector Identity for the Dirichlet Tesselation. Mathematical Proceedings of the Cambridge Philosophical Society, 87:151-155, 1980.

[6] N. Sukumar, B. Moran, and T. Belytschko. The Natural Element Method in Solid Mechanics. International Journal for Numerical Methods in Engineering, 43(5):839-887, 1998.

[7] O. C. Zienkiewicz and P. N. Godbolet. Flow of plastic and visco-plastic solids with special reference to extrusion and forming processes. International Journal for Numerical Methods in Engineering, 8:3-16, 1974. 